\title{
Erratum
}

\section{Magnetic Orbitals and Mechanisms of Exchange II. Superexchange J. Curély}

Monatsh Chem 136, 1013-1036

Concerning the definition of the intermediate cationic states (page 1023, Eq. (26)) the same letter symbols were printed for these new states as for the old states. Thus, care must be taken which states are meant in this part of the paper, especially in Eqs. (30), (34), (37), (43), (45), and (48).

In addition Eq. (31) should read:

$$
\mathcal{E}=-\left[(1-\alpha)^{2}+\beta^{2}\right](1-\delta) E-2 \beta t(1-\alpha) E
$$

And finally a factor 2 has been omitted and a wrong symbol has been used in Eq. (52); one must read:

$$
E_{0}^{U}=\mathcal{E}_{g}+\mathrm{E}_{u}+\frac{U-\gamma_{1}}{1-S^{2}}, \quad E_{1}^{U}=\mathcal{E}_{g}+\mathcal{E}_{u}+\frac{C-\gamma_{1}}{1-S^{2}}
$$

Verleger: Springer-Verlag GmbH, Sachsenplatz 4-6, 1201 Wien, Austria. - Herausgeber: Österreichische Akademie der Wissenschaften, Dr.-Ignaz-Seipel-Platz 2, 1010 Wien, und Gesellschaft Österreichischer Chemiker, Eschenbachgasse 9, 1010 Wien, Austria. - Redaktion: Getreidemarkt 9/163-OC, 1060 Wien, Austria. - Satz und Umbruch: Thomson Press Ltd., Chennai, India. Offsetdruck: Manz Crossmedia, 1051 Wien, Austria. - Verlagsort: Wien. - Herstellungsort: Wien. - Printed in Austria. 